\title{
Lung microbiome - a modern knowledge
}

\author{
DOROTA SIWICKA-GIEROBA, KATARZYNA CZARKO-WICHA
}

Department of Anesthesiology and Intensive Therapy, Medical University of Lublin, Lublin, Poland

\begin{abstract}
Recent studies have reported that commensal microorganisms are not just "passive occupants" but may play a crucial role in the immune system activation. It is well-known that in critically ill patients, the microbiome is modified and may be associated with the development of immunosuppression in sepsis, contributing to the development of acute renal injury, cardiovascular diseases, or more importantly, respiratory system disturbances. The conviction of lung sterility has gone down in history. The presence of characteristic gut microbiome, such as Bacteroidetes and Enterobacteriaceae, was demonstrated in lungs of critically ill patients. This bacteria's translocation, especially in ischemia-reperfusion injury, results in increased concentration of inflammation response markers and may play a pivotal role in the pathogenesis of respiratory system disturbances, including acute respiratory distress syndrome. Recent studies have shown that ischemia-reperfusion injury is often observed in intensive care units (ICUs) and predispose to microbiome disturbances that are strictly connected with immune system activation and epithelial damage. Potential effects of dysbiosis treatment are under highly activated investigation. Therefore, it is possible that microbiota-targeted therapy may constitute the future therapeutic path in ICUs.
\end{abstract}

Key words: lung microbiome, ARDS, VILI, ICUs, immune system cells.

(Cent Eur J Immunol 2020; 45 (3): 342-345)

\section{Introduction}

The human body is colonized by millions of microorganisms. It is believed that their number is at least as large as the number of cells forming the host organism [1]. Recent years have brought an increased technical ability to identify the human microbiome [2]. There are reports that commensal microorganisms are not just "passive occupants" since they have a significant impact on the immune response of their host [3]. It is known that in critically ill patients, the microbiome is modified [4, 5]. Disorders of microbiome may be associated with the development of immunosuppression in sepsis and contribute to the development of acute renal injury or cardiovascular diseases [6-8]. Therefore, it is important to assess the existence of possible dependencies between the microbiome and genetic or environmental factors in the aspect of immune response in critically ill patients [9].

There are numerous reports documenting the composition and role of the gut, skin, or vagina microbiome [10-12]. However, the role of commensal organisms living in the lungs is relatively unknown. In 2013, the number of 401,000 European people died from respiratory diseases, which accounted for $8 \%$ of all deaths recorded [13]. It can be assumed that the assessment of pulmonary microbiome's impact on the immune response of the host organism may indicate new therapeutic directions.

\section{Lung microbiome}

The conviction of lung sterility has gone down in history. By means of gastro-esophageal reflux or by microaspiration from the nasopharyngeal cavity, microorganisms constantly reach the alveoli [14]. The microbiome of healthy lungs consists mainly of bacteria from the Prevotella, Veillonella, and Fusobacterium species [15, 16]. Experimental studies have observed low density of pulmonary microbiome, with the value of the row $10^{3}-10^{5} \mathrm{CFU} / \mathrm{g}$ of a lung tissue [17]. Its current composition is determined by the mutual ratio of microorganism immigration to the airways, their elimination, and reproduction of bacterial colonies forming the microbiome [18]. However, the assessment of human microbiome in healthy individuals presents some difficulties. Non-intubated patients samples taken during bronchoscopy could be contaminated by bacterial flora of the nasopharynx. Furthermore, difficulties in acquiring microbial material, which represents a reliable composition of the pulmonary microbiome, concern intubated patients; the collected bronchoalveolar lavage fluid (BALF) often more closely indicates the microbiome of upper respiratory tract than the lungs. Only strongly invasive methods, such as an open lung biopsy allow to obtain a reliable material for testing [19]. However, experimental studies have shown that pulmonary microbiome is different from that in the oral cavity or the gut [4].

Correspondence: Dorota Siwicka-Gieroba, Department of Anesthesiology and Intensive Therapy, Medical University of Lublin, 8 Jaczewskiego St., 20-954 Lublin, Poland, e-mail: dsiw@wp.pl

Submitted: 17.12.2018; Accepted: 21.01.2019 
Animal studies indicate the role of forming respiratory airways microflora in the first few weeks of life in the development of a proper immune response of the organism, through the appearance of Helios (-) Treg cells and a reduction in the response to allergens. It results in lowering of susceptibility to the development of allergic airway inflammation in adulthood [20]. It has been shown that the early formation of the microbiome is responsible for the subsequent stability of upper respiratory tract microflora and the reduction of susceptibility to pulmonary infections [21]. Lungs of germ-free (GF) mice contain 2.5 times more invariant natural killer T cells (iNKT) than lungs of specific pathogen-free (SPF) mice. When the lungs of newborn GF mice were exposed to microorganisms being a standard component of normal microbiome, the number of iNKT cells became similar to that observed in SPF mice [22]. In the absence of commensal microorganisms, the number of infiltrating TH2 lymphocytes and eosinophils in the lungs increased [23]. Pulmonary microbiome, by modulating the expression of innate immunity genes, causes an increase in the concentration of IL-5, IL-10, IFN- $\gamma$, and CCL11. In the lungs of murine SPF newborns, the level of expression of PD-L1 on CD11b+ DCs and the frequency of FoxP3+ CD25+ Treg cells are higher [20]. The composition of microbiome also affects the TLR4-dependent response of pulmonary macrophages [24]. Moreover, microbiota modulate the production of antibacterial peptides (AMs) in the mucus and is responsible for inhibiting multiplication of bacteria and protecting the epithelium from these microorganisms [25]. It has been shown that in the absence of microbiome in the lungs or when its number is low, there is a decrease in the production of protective mucus [26]. The GF mice are more susceptible to pulmonary infections caused by Pseudomonas aeruginosa, Streptococcus pneumoniae, or Klebsiella pneumoniae [27, 28]. Additionally, the number of pulmonary alveoli is greater in the lungs with normal microbiome [17].

\section{Effect of gut microbiome on the lungs}

Gut microbiome is the largest and the most diverse group of commensal bacteria of the human body [29]. Microbiota shapes the immune response of the host reducing its susceptibility to the development of inflammation or infection and improves the integrity of intestinal wall [30-32]. Unfortunately, commensal bacteria are highly sensitive to environmental factors, including diet, antibiotics, allergens, and infectious pathogens, which can lead to microflora disorders, so-called "dysbiosis" [33]. Homeostasis disorders of the gut microbiome affect the distant organs, such as the brain, liver, vagina, or oral cavity [34]. Also, there is an evidence of interactions between the gut and the lungs in immunological aspect $[14,35]$. Gut dysbiosis has been shown to be associated with the development of infection and inflammatory response to the allergic back- ground in the respiratory system $[16,36]$. Both, the bacteria present in the gut and the products of their metabolism, may be the reason for immune stimulation within the lungs [37]. The lymph and/or blood pass through the immune signals from the originally sensitized gut to the lungs [38]. It is known, however, that signals can also be transmitted in the opposite direction. The dysbiosis of pulmonary microbiome caused by tracheal administration of lipopolysaccharide leads to disorders in homeostasis of the gut microbiome [39]. Experimental studies documented the influence of pneumonia with etiology of a multi resistant drug to Staphylococcus aureus or Pseudomonas aerugino$s a$ on the occurrence of gut damage [40]. For this reason, the reciprocal bi-directional relationship of these two local microbiomes is called the "gut-lung axis".

\section{Lung microbiome in critically ill patients}

As a result of imbalance between the elimination and inflow of bacteria to the lungs during the disease, the composition of pulmonary microbiome is disturbed [41]. Disorders of the physiological flora of the lungs in asthma or chronic obstructive pulmonary disease have been documented [42, 43]. However, it is known that pulmonary dysbiosis also occurs in critically ill patients, in whom the primary pathology did not have a pulmonary source. The etiology of lung dysbiosis in critically ill patients is complex. First of all, such patients often undergo antibiotic therapies [44]. Schuijt et al. showed that in mice that received antibiotics, lung inflammation, organ damage, and mortality associated with Streptococcus pneumoniae infection was higher than in the control group [45]. Wienhold et al. documented that a wide-spectrum antibiotic therapy, which leads to microbial disturbances in experimental animals and ventilation with high tidal volume, increases the susceptibility to ventilatory-induced lung injury (VILI) development [46]. Secondly, endotracheal intubation and mechanical lung ventilation favor the phenomenon of micro-aspiration and impair the mechanisms of natural cleansing of the airways, which promotes the dominance of opportunistic pathogens [15, 47]. In addition, the ongoing inflammatory process in septic patients or the nutrient-rich edema found in acute respiratory distress syndrome (ARDS) are responsible for the modification of metabolic and physicochemical conditions of the lungs $[48,49]$. Furthermore, the presence of bacteria characteristic for the gut microbiome, such as Bacteroidetes and Enterobacteriaceae, has been demonstrated in critically ill lungs, which results in an increase in the concentration of inflammation response markers and may play an active role in the pathogenesis of ARDS [48, 50, 51]. Recent studies presented that ischemia-reperfusion injury, often observed in ICUs, predispose to microbiota disturbances strictly connected with immune system activation and epithelial damage. Elevated growth of Enterobacteriaceae 
promotes and perpetuates inflammation and trauma processes [52]. Hammer et al. showed that some elements of immune system can protect epithelial barrier during burn trauma. IL-22 and STAT3 signaling regulate the epithelial barrier, intestinal microbiome, and may prevent translocation of Enterobacteriaceae [53]. Importantly, interleukin 22 reduces inflammation in lungs. During mechanical ventilation, $\mathrm{pH}$, free-radicals production, or oxygen tension changes, and alveolars are the potential target of pathogens growth. Some studies showed that intestinal typical bacteria are detected in the lung, serum, and mesenteric cells after ischemia-reperfusion injury. Intestine microbiome use activation of TLR 2, TLR 4, and myeloid differentiation primary-response 88 (MyD88) to tissue damage via nitric oxide synthase (iNOS) and reactive oxygen species (ROS) production.

A meta-analysis conducted by Manzanares et al. evaluating the effects of probiotics in the treatment of patients hospitalized in intensive care units (ICUs) showed a reduction in the incidence of ventilatory-associated pneumonia (VAP); however, there was no effect on mortality or length of hospitalization [54]. Therefore, it is possible that microbiota-targeted therapy may constitute the future therapeutic direction in ICUs.

\section{The authors declare no conflict of interest.}

\section{References}

1. Sender R, Fuchs S, Milo R (2016): Revised Estimates for the Number of Human and Bacteria Cells in the Body. PLoS Biol 14: e1002533.

2. Sender R, Fuchs S, Milo R (2016): Are We Really Vastly Outnumbered? Revisiting the Ratio of Bacterial to Host Cells in Humans. Cell 164: 337-340.

3. Schirmer M, Smeekens SP, Vlamakis H, et al. (2016): Linking the Human Gut Microbiome to Inflammatory Cytokine Production Capacity. Cell 167: 1125-1136.e8.

4. Dickson RP (2018): The Lung Microbiome and ARDS. It Is Time to Broaden the Model. Am J Respir Crit Care Med 197: 549-551.

5. Ojima M, Motooka D, Shimizu K, et al. (2016): Metagenomic Analysis Reveals Dynamic Changes of Whole Gut Microbiota in the Acute Phase of Intensive Care Unit Patients. Dig Dis Sci 61: 1628-1634.

6. Krezalek MA, DeFazio J, Zaborina O, et al. (2016): The Shift of an Intestinal "Microbiome" to a "Pathobiome" Governs the Course and Outcome of Sepsis Following Surgical Injury. Shock 45: 475-482.

7. Andrade-Oliveira V, Amano MT, Correa-Costa M, et al. (2015): Gut Bacteria Products Prevent AKI Induced by IschemiaReperfusion. J Am Soc Nephrol 26: 1877-1888.

8. Tang WHW, Kitai T, Hazen SL (2016): Gut Microbiota in Cardiovascular Health and Disease. Circ Res 120: 1183-1196.

9. Gotts JE, Matthay MA (2016): Sepsis: pathophysiology and clinical management. BMJ 353: i1585.

10. Kamada N, Seo S-U, Chen GY, Núńez G (2013): Role of the gut microbiota in immunity and inflammatory disease. Nat Rev Immunol 13: 321-335.
11. Scharschmidt TC (2017): Establishing Tolerance to Commensal Skin Bacteria. Dermatol Clin 35: 1-9.

12. Nunn KL, Forney LJ (2016): Unraveling the Dynamics of the Human Vaginal Microbiome. Yale J Biol Med 89: 331-337.

13. Eurostat statistics explained. https://ec.europa.eu/eurostat/statistics-explained/index.php/Main_Page/pl (access: 7.12.2018).

14. Budden KF, Gellatly SL, Wood DLA, et al. (2017): Emerging pathogenic links between microbiota and the gut-lung axis. Nat Rev Microbiol 15: 55-63.

15. Kelly BJ, Imai I, Bittinger K, et al. (2016): Composition and dynamics of the respiratory tract microbiome in intubated patients. Microbiome 4: 7.

16. Shukla SD, Budden KF, Neal R, Hansbro PM (2017): Microbiome effects on immunity, health and disease in the lung. Clin Transl Immunol 6: e133.

17. Remot A, Descamps D, Noordine M-L, et al. (2017): Bacteria isolated from lung modulate asthma susceptibility in mice. ISME J 11: 1061-1074.

18. Dickson RP, Martinez FJ, Huffnagle GB (2014): The role of the microbiome in exacerbations of chronic lung diseases. Lancet 384: 691-702.

19. Yu G, Gail MH, Consonni D, et al. (2016): Characterizing human lung tissue microbiota and its relationship to epidemiological and clinical features. Genome Biol 17: 163.

20. Gollwitzer ES, Saglani S, Trompette A, et al. (2014): Lung microbiota promotes tolerance to allergens in neonates via PD-L1. Nat Med 20: 642-647.

21. Biesbroek G, Tsivtsivadze E, Sanders EAM, et al. (2014): Early Respiratory Microbiota Composition Determines Bacterial Succession Patterns and Respiratory Health in Children. Am J Respir Crit Care Med 190: 1283-1292.

22. Olszak T, An D, Zeissig S, et al. (2012): Microbial Exposure During Early Life Has Persistent Effects on Natural Killer T Cell Function. Science 336: 489-493.

23. Herbst T, Sichelstiel A, Schär C, et al. (2011): Dysregulation of Allergic Airway Inflammation in the Absence of Microbial Colonization. Am J Respir Crit Care Med 184: 198-205.

24. Segal LN, Rom WN, Weiden MD (2014): Lung Microbiome for Clinicians. New Discoveries about Bugs in Healthy and Diseased Lungs. Ann Am Thorac Soc 11: 108-116.

25. Gallo RL, Hooper LV (2012): Epithelial antimicrobial defence of the skin and intestine. Nat Rev Immunol 12: 503-516.

26. Yun Y, Srinivas G, Kuenzel S, et al. (2014): Environmentally Determined Differences in the Murine Lung Microbiota and Their Relation to Alveolar Architecture. PLoS One 9: e113466.

27. Brown RL, Sequeira RP, Clarke TB (2017): The microbiota protects against respiratory infection via GM-CSF signaling. Nat Commun 8: 1512.

28. Fox AC, McConnell KW, Yoseph BP, et al. (2012): The Endogenous Bacteria Alter Gut Epithelial Apoptosis and Decrease Mortality Following Pseudomonas aeruginosa Pneumonia. Shock 38: 508-514.

29. Backhed F, Ley RE, Sonnenburg JL, et al. (2005): Host-Bacterial Mutualism in the Human Intestine. Science 307: 1915-1920.

30. Gensollen T, Iyer SS, Kasper DL, Blumberg RS (2016): How colonization by microbiota in early life shapes the immune system. Science 352: 539-544.

31. Mazmanian SK, Round JL, Kasper DL (2008): A microbial symbiosis factor prevents intestinal inflammatory disease. Nature 453: 620-625.

32. Natividad JMM, Verdu EF (2013): Modulation of intestinal barrier by intestinal microbiota: Pathological and therapeutic implications. Pharmacol Res 69: 42-51. 
33. Thaiss CA, Zmora N, Levy M, Elinav E (2016): The microbiome and innate immunity. Nature 535: 65-74.

34. Shreiner AB, Kao JY, Young VB (2015): The gut microbiome in health and in disease. Curr Opin Gastroenterol 31: 69-75.

35. Bingula R, Filaire M, Radosevic-Robin N, et al. (2017): Desired Turbulence? Gut-Lung Axis, Immunity, and Lung Cancer. J Oncol 2017: 1-15.

36. Russell SL, Gold MJ, Willing BP, et al. (2013): Perinatal antibiotic treatment affects murine microbiota, immune responses and allergic asthma. Gut Microbes 4: 158-164.

37. Hauptmann M, Schaible UE (2016): Linking microbiota and respiratory disease. FEBS Lett 590: 3721-3738.

38. He Y, Wen Q, Yao F, et al. (2017): Gut-lung axis: The microbial contributions and clinical implications. Crit Rev Microbiol 43: 81-95.

39. Sze MA, Tsuruta M, Yang S-WJ, et al. (2014): Changes in the bacterial microbiota in gut, blood, and lungs following acute LPS instillation into mice lungs. PLoS One 9: e111228.

40. Perrone EE, Jung E, Breed E, et al. (2012): Mechanisms of Methicillin-Resistant Staphylococcus aureus Pneumonia-Induced Intestinal Epithelial Apoptosis. Shock 38: 68-75.

41. Dickson RP, Huffnagle GB (2015): The Lung Microbiome: New Principles for Respiratory Bacteriology in Health and Disease. PLoS Pathog 11: e1004923.

42. Hooks KB, O'Malley MA (2017): Dysbiosis and Its Discontents. MBio 8: e01492-17.

43. Pragman AA, Kim HB, Reilly CS, et al. (2012): The Lung Microbiome in Moderate and Severe Chronic Obstructive Pulmonary Disease. Taube C, ed. PLoS One 7: e47305.

44. Zarb P, Coignard B, Griskeviciene J, et al. The European Centre for Disease Prevention and Control (ECDC) Pilot Point Prevalence Survey of Healthcare-Associated Infections and Antimicrobial Use. www.eurosurveillance.orghttp://www.eurosurveillance. org/ViewArticle.aspx?ArticleId=20316 (access: December 7, 2018).

45. Schuijt TJ, Lankelma JM, Scicluna BP, et al. (2016): The gut microbiota plays a protective role in the host defence against pneumococcal pneumonia. Gut 65: 575-583.

46. Wienhold S-M, Macrě M, Nouailles G, et al. (2018): Ventilatorinduced lung injury is aggravated by antibiotic mediated microbiota depletion in mice. Crit Care 22: 282.

47. Nseir S, Zerimech F, Jaillette E, et al. (2011): Microaspiration in intubated critically ill patients: diagnosis and prevention. Infect Disord Drug Targets 11: 413-423.

48. Dickson RP, Singer BH, Newstead MW, et al. (2016): Enrichment of the lung microbiome with gut bacteria in sepsis and the acute respiratory distress syndrome. Nat Microbiol 1: 16113.

49. Dickson RP, Erb-Downward JR, Huffnagle GB (2013): The role of the bacterial microbiome in lung disease. Expert Rev Respir Med 7: 245-257.

50. Dickson RP (2016): The microbiome and critical illness. Lancet Respir Med 4: 59-72.

51. Panzer AR, Lynch SV, Langelier C, et al. (2018): Lung Microbiota Is Related to Smoking Status and to Development of Acute Respiratory Distress Syndrome in Critically Ill Trauma Patients. Am J Respir Crit Care Med 197: 621-631.

52. Lupp C, Robertson M, Wickham M, et al. Host-mediated inflammation disrupts the intestinal microbiota and promotes the overgrowth of Enterobacteriaceae. https://www.sciencedirect.com/science/article/pii/S1931312807001576 (access: December 2, 2018).
53. Hammer AM, Morris NL, Cannon AR, et al. (2017): Interleukin-22 Prevents Microbial Dysbiosis and Promotes Intestinal Barrier Regeneration Following Acute Injury. Shock 48: 657-665.

54. Manzanares W, Lemieux M, Langlois PL, Wischmeyer PE (2016): Probiotic and synbiotic therapy in critical illness: a systematic review and meta-analysis. Crit Care 20: 262. 